\title{
The future of combination therapies in advanced melanoma
}

\author{
Christoph Hoeller (D)
}

Received: 17 June 2020 / Accepted: 20 July 2020 / Published online: 14 August 2020

(C) The Author(s) 2020

\begin{abstract}
Summary The combination of Cytotoxic T-Lymphozyte Antigen-4 (CTLA-4) and Programmed death1 (PD-1) antibodies and the combination of BRAF and MEK inhibitors are the current clinical standards for combination immune and targeted therapy for melanoma, respectively. The success of these therapies has stimulated research into novel drug combinations for melanoma, of which a large majority are based on combination with PD-1 or PD-Ligand 1 (PD-L1) blocking drugs. Thus, the aim is to provide an overview of the most important combination strategies in late stage clinical development and an outlook on drug combinations in early development that might enter larger clinical trials within the next few years.
\end{abstract}

Keywords Skin cancer · Immunotherapy · BRAF . Intralesional therapy · Vaccine

\section{Introduction}

The combination of ipilimumab (Ipi) and nivolumab (Nivo) and the combination of BRAF and MEK inhibitors are current standards for combination therapies in immunotherapy and targeted therapy of melanoma [1, 2]. Recent data from pivotal studies have demonstrated that roughly up to $35 \%$ (for BRAF/MEK inhibitors) and 50\% (for Ipi/Nivo) of firstline patients treated with these combinations are still alive after 5 years. While this is a significant gain over the past standard of chemotherapy, it means that at least half of these patients will not achieve a long-term benefit. Several new combinations that

\section{Hoeller $(\bowtie)$}

Department of Dermatology, Medical University of Vienna,

Waehringer Guertel 18-20, 1090 Vienna, Austria

christoph.hoeller@meduniwien.ac.at aim to further improve these numbers are currently undergoing late-stage clinical development.

\section{Targeted inhibitors and PD-1/PD-L1 blocking antibodies}

The clinical rationale for combining targeted and immunotherapy is to utilize the rapid onset of response and high response rate of BRAF/MEK inhibitors and the longer duration of benefit seen in patients responding to checkpoint inhibitors (CPI). In addition, a preclinical rationale is that early during targeted treatment an influx of T-cells into the tumor is observed which could be augmented by adding a CPI [3]. The combination of vemurafenib and cobimetinib with the PD-L1 blocking antibody atezolizumab (NCT02908672, Trilogy) as well as the combination of dabrafenib and trametinib with the PD-1 blocking antibody spartalizumab (NCT02967692, Combi-I) are currently being tested in phase III trials in patients with BRAF mutations and in comparison to the respective BRAF/MEK inhibitor combination. Data from Trilogy presented at AACR showed a median investigator assessed progression-free survival (PFS) of 15.1 vs. 10.6 months $(P=0.0249)$ in favor of the triplet arm. However, no difference in response rate was seen between the two arms, especially no higher number of complete responses (CRs) was observed with the triplet. Data on overall survival (OS) was immature with some early separation at the 24 month time point [4]. For Combi-I only data from an early safety and biomarker cohort has been presented but spiked interest with a high CR rate of $44 \%$ [5]. Phase III data from Combi-I is expected later this year.

The main issue with these triplet trials is on the one hand that the comparator arm of BRAF/MEK inhibition is not the recommended first-line therapy for unresectable metastatic melanoma in major guidelines. 
Independent of the mutational status, this is nowadays a PD-1 based CPI regimen. On the other hand, the PFS landmarks in Trilogy of $54 \%$ investigator assessed PFS at 12 months and $43.6 \%$ at 18 months are not better than what was shown to be possible with the combination of Ipi and Nivo [6]. Especially since Trilogy had only a bit more than $30 \%$ of patients with elevated LDH and thus a rather positively skewed population, this raises the question whether it would be really an advantage to use both the immunotherapy and the targeted therapy option in first-line rather than saving targeted therapy as an ideal second-line treatment in the case of CPI failure.

\section{Novel checkpoint inhibitor combinations}

Currently several alternative immune check points like Tim-3, Tigit, Vista LAG-3 and others are tested, mostly in combination with PD1 inhibitors in clinical trials. While the majority are in early phases of clinical development, the LAG-3 antibody relatlimab is currently being tested in combination with nivolumab against nivolumab alone in the first-line treatment of patients with unresectable metastatic melanoma (NCT03470922). While no data from this trial is available, relatlimab is also being tested in an ongoing phase II trial in patients after progression on or following PD-1 antibody treatment (NCT01968109). An early assessment from this trial presented at ESMO in 2017 did show an ORR with relatlimab and nivolumab of $11.5 \%$ which increased to $18 \%$ in patients with $>1 \%$ of LAG-3 expression on the tumor, indicating that such a therapy could in the future be selected based on a biomarker [7].

\section{Combination therapies with cytokines}

In the early days of checkpoint inhibition, cytokines like IL-2 or GM-CSF were combined with ipilimumab in early phase clinical trials, but none of these combinations was developed until approval. Bempegaldesleukin (NKTR214/Bempeg) is a PEGylated version of $1 \mathrm{~L}-2$ that is delivered as a 6-PEG prodrug from which the active 2-PEG and 1-PEG forms are released. The active forms exhibit a relative selectivity for Il$2 \mathrm{R} \beta \gamma$ which is preferentially expressed on cytotoxic $\mathrm{T}$ and NK-cells over Il2-R $\alpha \beta \gamma$ preferentially active on regulatory T-cells. In the phase I/II trial Pivot 02 a combination of nivolumab with bempeg did show an encouraging CR rate of $34 \%$ in first-line patients with stage IV melanoma. In contrast to classic highdose IL-2, bempeg can be administered on an outpatient basis as high-grade side effects associated with a vascular leak syndrome have not been observed [8]. This combination is currently tested in a phase III trial against nivolumab alone (NCT03635983).

\section{Combinations with intralesional drugs}

Talimogene laherparepvec (TVEC) is a modified herpes-simplex type I virus that replicates preferentially in tumor cells and expresses human GM-CSF. In Europe TVEC is approved for intralesional treatment of melanoma patients with injectable, superficial lesions in unresectable stage III or stage IV M1a. The combination of viral lysis of tumor cells with an immune induction by pathogen-induced signals as well as by the expression of hGMCSF has been demonstrated to induce regression of injected as well as of uninjected lesions. The quintessential action of TVEC as a form of personalized in situ tumor-vaccine then spiked interest in using TVEC together with CPIs. Combinations with ipilimumab as well as pembrolizumab were explored in phase II protocols. In a randomized phase II study, the combination of TVEC + Ipi was compared to Ipi alone and the study met its primary endpoint with an ORR of $39 \%$ over $18 \%$ in favor of the combination arm. This was not only limited to injected lesions but did also extend to regression of uninjected visceral lesions which was observed in $52 \%$ of patients in the combination arm vs. $23 \%$ of patients in the Ipi arm. PFS was not significantly different, although numerically higher in the combination arm, where Ipi was only started after 5 weeks of initial TVEC treatment [9]. While OS for the whole study is not yet mature, the number of CRs in each treatment arm-21.4\% vs. $6 \%$ for TVEC + Ipi and Ipi, respectively-was demonstrated to be associated with an improvement in longterm survival [10].

Translational proof of principle was provided by a single arm phase II study that combined TVEC with pembrolizumab with a 6 week run-in phase of TVEC before adding pembrolizumab. Confirmed objective response rate was $62 \%$, with a complete response rate of $33 \%$ per immune-related response criteria. An initial increase of intratumoral CD8+ T-cells following administration of TVEC that was augmented by the addition of pembrolizumab, and the level of response was independent of the number of CD8+ T-cells at baseline [11].

The combination of TVEC and pembrolizumab is currently being tested vs. pembrolizumab alone in the randomized Masterkey-265 trial (NCT02263508).

Tilsotolimod (IMO-2125) is a synthetic oligonucleotide which binds to TLR9 and activates antigen presentation of dendritic cells and macrophages with subsequent proliferation of antigen specific cytotoxic $\mathrm{T}$ lymphocytes (CD8+ T-cells) in both injected and uninjected tumors. Tilsotolimod was tested in a phase I/II study in combination with ipilimumab in $26 \mathrm{PD}-1$ refractory melanoma patients (NCT02644967). An ORR of 38\% with 9.5\% CRs was observed which exceeds the expected response rate to ipilimumab alone and the combination was well tolerated [12]. A phase III study with this combination 
in comparison to ipilimumab alone in PD-1 refractory patients is ongoing (NCT03445533).

PV-10 is an injectable formulation of rose bengal disodium, a water-soluble xanthene dye otherwise used in topical ophthalmic diagnostics. PV-10 was shown to induce tumor-specific interferon $\gamma$-release and tumor-cell apoptosis. Based on response rates above $50 \%$ in injected and uninjected metastases in early clinical studies [13] PV-10 is currently being tested in an open label randomized controlled trial in combination with the PD-1 inhibitor pembrolizumab (NCT02288897).

\section{Combinations in early clinical development}

Many other combinations are being tested in early clinical protocols that also include melanoma patients, and are mostly based on a PD-1 inhibitor backbone. Based on different preclinical rational, which has been reviewed more extensively elsewhere, they target diverse aspects of the immune response [14].

Glucocorticoid-induced TNFR-related protein (GITR), a member of the TNF receptor superfamily is expressed at high levels on regulatory T-cells. Drugs blocking GITR have been shown to decrease the number of regulatory T-cells, while activating CD8+ T-cells [15]. Different inhibitors of GITR are currently being tested in combination with PD-1 inhibitors alone or in combination with ipilimumab in phase I/II trials.

Stimulator of interferon genes (STING) is an intracellular receptor that controls the transcription of immune response genes like type I interferons, proinflammatory cytokines or chemokines typically in response to triggers of the innate immune system, including signals from dendritic cells in response to necrotic tumor cells. STING knock-out mice have been shown to have an increased susceptibility to tumors and deactivation of STING prevented the activity of checkpoint inhibitors in preclinical models [16]. Recently stimulators of STING have been tested in early clinical trials and are currently being developed in combination with PD-1 blocking drugs.

The combination of the HDAC inhibitor entinostat was tested in combination with pembrolizumab in 53 patients who had previously progressed on or after PD-1 based therapy in the ENCORE-601 study (NCT02437136). The rationale behind the use of an HDAC inhibitor is to inhibit regulatory T-cells as well as myeloid-derived suppressor cells in the tumor microenvironment. The ORR was $19 \%$ with $1 \mathrm{CR}$ and 5 PRs [17]. This combination is currently also being tested in patients with metastatic uveal melanoma (NCT02697630).

Adenosine is produced at high levels in the hypoxic tumor microenvironment and exerts immunosuppressive functions through the $\mathrm{A}_{2 \mathrm{~A}}$ receptor expressed on various immune cells [18]. Adenosine is released from ATP through conversion by CD39 and CD73. Inhibitors of CD73 and the A2A receptor are currently in clinical development in combination with PD-1 or PD-L1 blocking antibodies in solid tumors, including melanoma.

Several personalized, neoepitope-specifc RNA vaccines are under clinical development and are also combined with PD-1 inhibitors. These vaccines are based on analysis of the tumor-specific set of mutations (the "mutanome"). From these mutations altered protein structures-so-called "neoepitopes"-can be predicted and sorted for their possible immune reactivity. A set of such patient-specific neoepitopes is then injected into lymph nodes or infused in the form of a stabilized mRNA structure leading to in loco translation of the immunogenic protein, thus, leading to induction of a tumor-specific immune response [19].

\section{Discussion}

A wide range of new combinations is developed for the therapy of melanoma. Currently the majority of these trials are addressing first-line patients with unresectable metastatic melanoma. These trials do however face the challenge to demonstrate an equal or higher efficacy than the combination of ipilimumab and nivolumab, the current benchmark for efficacy in melanoma therapy.

One way to overcome this obstacle would be to demonstrate superiority in a biomarker-defined subset of patients. While PD-L1 has been repeatedly shown to lack value as a biomarker in melanoma patients, expression of other target proteins (e.g., LAG-3) might become useful biomarkers for some therapies. Specific immune signatures that show deficiencies in the tumor-immune response that would allow to rationally choose combinations addressing specific defects would be an alternative strategy. Intriguing concepts, like the cancer immunogram [20], have provided theoretical insight into the necessary components of the tumor-immune response and several assays like the interferon-gamma signature, mutational load or spatial distribution of immune cells are currently under development. Together with other biomarkers, e.g. the gut microbiome, they might in the future guide treatment decisions [21].

The biggest challenge we face today in melanomatherapy is however patients who have already progressed on PD-1-based therapies, especially if they lack a BRAF mutation. While encouraging signals in this field come from some of the novel combinations described, only a minority of these studies address this burning question. The main difficulty in designing trials specifically for this patient population is that despite the increasing amount of information on possible resistance mechanisms we do-similar to the first-line decision-still lack established markers that would allow us to point out a specific resistance mech- 
anism for a therapeutic decision. Therefore, drugs like HDAC inhibitors that can target several resistance mechanisms at the same time [22] might at the moment be specifically interesting in this setting.

At the same time some of these combinations have already moved into the field of adjuvant and neoadjuvant therapy and might, similar to PD-1 inhibitors and BRAF/MEK inhibitors, provide benefit to a larger number of patients in these earlier tumor stages.

In summary, the number of new combinations under development for melanoma raises the hope that the number of patients achieving a therapeutic benefit will increase with these therapies. At the same time the question on the optimal algorithm of firstand later-line therapies and the search for biomarkers to guide these decisions are still under development and will require ongoing research efforts in the coming years.

\section{Take home message}

Novel combination therapies will increase the number of therapeutic options available for the treatment of metastatic melanoma and will help us to increase the number of patients acheiving a long-term clinical benefit.

Funding Open access funding provided by Medical University of Vienna.

Conflict of interest C. Hoeller declares the following possible conflict of interests: speaker for Amgen, BMS, MSD, Novartis and Roche. Consultancy for Amgen, Astra Zeneca, BMS, Inzyte, MSD, Novartis, Pierre Fabre, Roche. Research support from Amgen.

Open Access This article is licensed under a Creative Commons Attribution 4.0 International License, which permits use, sharing, adaptation, distribution and reproduction in any medium or format, as long as you give appropriate credit to the original author(s) and the source, provide a link to the Creative Commons licence, and indicate if changes were made. The images or other third party material in this article are included in the article's Creative Commons licence, unless indicated otherwise in a credit line to the material. If material is not included in the article's Creative Commons licence and your intended use is not permitted by statutory regulation or exceeds the permitted use, you will need to obtain permission directly from the copyright holder. To view a copy of this licence, visit http://creativecommons.org/licenses/by/4.0/.

\section{References}

1. Garbe C, Amaral T, Peris K, Hauschild A, Arenberger P, Bastholt L, et al. European consensus-based interdisciplinaryguidelinefor melanoma. Part2: Treatment-Update 2019. Eur JCancer. 2020;126:159-77.

2. Michielin O, van Akkooi ACJ, Ascierto PA, Dummer R, Keilholz U. Cutaneous melanoma: ESMO Clinical Practice Guidelines for diagnosis, treatment and follow-updagger. Ann Oncol. 2019;30(12):1884-901. clinicalguidelines@esmo.orgEGCEa.
3. Ascierto PA, Dummer R. Immunological effects of BRAF+MEK inhibition. OncoImmunology. 2018;7(9): e1468955.

4. Gutzmer R, Stroyakovskiy D, Gogas H, Robert C, Lewis K, Protsenko S, et al. Atezolizumab, vemurafenib, and cobimetinib as first-line treatment for unresectable advanced BRAF(V600) mutation-positive melanoma (IMspire150): primary analysis of the randomised, double-blind, placebo-controlled, phase 3 trial. Lancet. 2020;395(10240):1835-44.

5. Long GV, Lebbe C, Atkinson V, Mandala M, Nathan P, Arance A, et al. The anti-PD-1 antibody spartalizumab in combination with dabrafenib and trametinib in advanced BRAF V600-mutant melanoma: efficacy and safety findings from parts 1 and 2 of the Phase IIICOMBI-itrial. JClin Oncol. 2020;38. suppl; abstr 10028. https://doi.org/10.1200/JCO. 2020.38.15_suppl.10028.

6. Larkin J, Chiarion-Sileni V, Gonzalez R, GrobJJ, Rutkowski P, Lao CD, et al. Five-year survival with combined Nivolumab and Ipilimumab in advanced melanoma. N Engl J Med. 2019;381(16):1535-46.

7. Ascierto et al., ESMO 2017, Abstract LBA18

8. Hurwitz ME, Cho DC, Balar AV, Curti BD, Siefker-Radtke AO, Sznol M, et al. Baseline tumor-immune signatures associated with response to bempegaldesleukin (NKTR-214) and nivolumab. J Clin Oncol. 2019;37. suppl; abstr 2623. https://doi.org/10.1200/JCO.2019.37.15_suppl.2623.

9. Chesney J, Puzanov I, Collichio F, Singh P, Milhem MM, Glaspy J, et al. Randomized, open-label phase II study evaluating the efficacy and safety of Talimogene Laherparepvec in combination with Ipilimumab versus Ipilimumab alone in patients with advanced, unresectable melanoma. J Clin Oncol. 2018;36(17):1658-67.

10. Chesney J, Puzanov I, Collichio F, Milhem MM, Hauschild A, Yi M, et al. Association between complete response and survival in advanced melanoma treated with talimogene laherparepvec (T-VEC) plus ipilimumab (ipi). J Clin Oncol. 2020;38. suppl; abstr 10029. https://doi.org/10.1200/JCO. 2020.38.15_suppl.10029.

11. Ribas A, Dummer R, Puzanov I, VanderWalde A, Andtbacka RHI, Michielin O, et al. Oncolytic virotherapy promotes intratumoral T cell infiltration and improves antiPD-1 immunotherapy. Cell. 2017;170(6):1109-1119 e10.

12. Diab A, Rahimian S, Haymaker CL, Bernatchez C, Andtbacka RHI, James M, et al. A phase 2 study to evaluate the safety and efficacy of Intratumoral (IT) injection of the TLR9 agonist IMO-2125 (IMO) in combination with ipilimumab (ipi) in PD-1 inhibitor refractory melanoma. J Clin Oncol. 2018;36. suppl; abstr 9515. https://doi.org/10.1200/JCO. 2018.36.15_suppl.9515.

13. Thompson JF, Agarwala SS, Smithers BM, Ross MI, Scoggins CR, Coventry BJ, et al. Phase 2 study of intralesional PV-10 in refractory metastatic melanoma. Ann Surg Oncol. 2015;22(7):2135-42.

14. Mandala M, Rutkowski P. Rational combination of cancer immunotherapy in melanoma. Virchows Arch. 2019;474(4):433-47.

15. Knee DA, Hewes B, Brogdon JL. Rationale for anti-GITR cancer immunotherapy. Eur J Cancer. 2016;67:1-10.

16. Villanueva MT. STINGing systemically. Nat Rev Drug Discov. 2019;18(1):15.

17. Sullivan O et al, AbstractCT072, AACR 2019

18. Leone RD, Emens LA. Targeting adenosine for cancer immunotherapy. J Immunother Cancer. 2018;6(1):57.

19. Sahin U, Derhovanessian E, Miller M, Kloke BP, Simon P, Lower M, et al. Personalized RNA mutanome vaccines mo- 
bilize poly-specific therapeutic immunity against cancer. Nature. 2017;547(7662):222-6.

20. Blank CU, Haanen JB, Ribas A, Schumacher TN. Cancer immunology. The "cancer immunogram". Science. 2016;352(6286):658-60.

21. Twitty CG, Huppert LA, Daud AI. Prognostic biomarkers for melanomaimmunotherapy. CurrOncolRep. 2020;22(3):25.

22. Yeon M, Kim Y, Jung HS, Jeoung D. Histone Deacetylase Inhibitors to Overcome Resistance to Targeted and Immuno Therapy in Metastatic Melanoma. Front Cell Dev Biol. 2020;8:486.
Publisher's Note Springer Nature remains neutral with regard to jurisdictional claims in published maps and institutional affiliations.

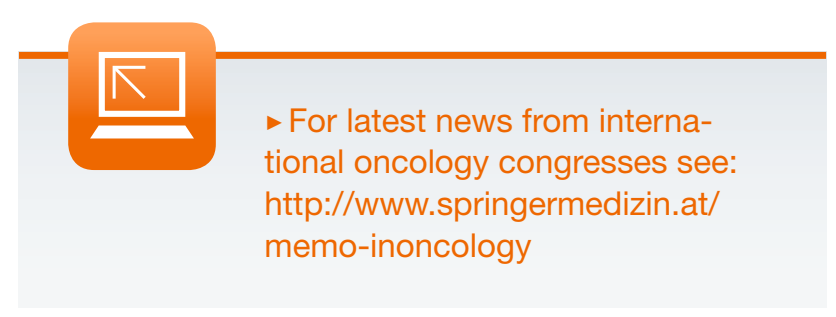

